\title{
Enhancing Statin Adherence Using a Motivational Interviewing Intervention and Past Adherence Trajectories in Patients with Suboptimal Adherence
}

\author{
Susan M. Abughosh, PhD; Aisha Vadhariya, PhD; Michael L. Johnson, PhD; \\ E. James Essien, MD, DrPH; Tara W. Esse, PharmD, BCACP; Omar Serna, PharmD, BCACP; \\ Esteban Gallardo, PharmD; Susan H. Boklage, MS, MPH; Jeannie Choi, PharmD; \\ Marcia M. Holstad, PhD, FNP-BC, FAAN; and Marc L. Fleming, PhD, MPH, RPh
}

\begin{abstract}
BACKGROUND: Statins have been shown to be effective in reducing the occurrence of cardiovascular (CV) events and are widely prescribed for the risk reduction of CV diseases and recurrent CV events. However, poor adherence prevents some patients from receiving the maximum benefit of the therapy. Motivational interviewing ( $\mathrm{Mol}$ ) is a patient-centered collaborative approach that can be used to improve medication adherence. Groupbased trajectory modeling depicts patterns of adherence over time and may help tailor the Mol intervention to further enhance adherence.
\end{abstract}

OBJECTIVE: To assess the effect of a phone-based Mol intervention tailored by patients' past adherence trajectory in improving adherence to statins among patients in a Medicare Advantage prescription drug plan (MAPD). METHODS: Patients continuously enrolled in an MAPD from 2013 to 2017 with a statin prescription between January and June 2015 to allow 2 years of pre-index period and 1 year of follow-up were included in the study. Adherence to statins was measured monthly during the 1-year follow-up as proportion of days covered (PDC) and incorporated into a group-based trajectory model to provide 4 distinct patterns of adherence: adherent, rapid decline, gradual decline, and gaps in adherence. Patients in the 3 nonadherent groups were randomized to either control or intervention. The intervention was an initial counseling call and up to 2 monthly followup calls by pharmacy students trained in Mol, providing education consistent with a previously identified pattern of use. Refill data at 6 months post-intervention were evaluated to examine the intervention's effect on $P D C$, as continuous and dichotomized as $P D C \geq 0.8$, as well as discontinuation. Multivariable regression adjusted for baseline demographics, clinical characteristics, and past adherence trajectory.

RESULTS: There were 152 patients included in the analysis who received Mol phone calls and 304 randomly selected controls. Mean PDC for the intervention group $(0.67 \pm 0.3)$ was significantly higher than the control $(0.55 \pm 0.4 ; P<0.001)$. The intervention group was also less likely to discontinue $(\mathrm{OR}=0.38 ; 95 \% \mathrm{Cl}=0.19-0.76)$ and more likely to be adherent in the linear regression model $(\beta=12.4 ; P<0.001)$ as well as in the logistic regression model $(\mathrm{OR}=1.87 ; 95 \% \mathrm{Cl}=1.18-2.95)$. Previous adherence trajectories were significantly associated with adherence in the follow-up.

CONCLUSIONS: Patients who received the Mol intervention were more likely to be adherent and less likely to discontinue the statin in the 6 months follow-up compared with controls. Future research can identify other approaches to tailor interventions and expand the intervention to other languages. This intervention may also prove valuable to improve adherence to other medications for chronic and asymptomatic diseases.

J Manag Care Spec Pharm. 2019;25(10):1053-62

Copyright $\odot 2019$, Academy of Managed Care Pharmacy. All rights reserved.

\section{What is already known about this subject}

Suboptimal adherence to statins is a prevalent issue affecting cardiovascular outcomes.

Group-based trajectory modeling can characterize adherence behavior patterns over a defined time period.

Motivational interviewing interventions have demonstrated an improvement in patient adherence.

\section{What this study adds}

Patient characteristics that predict a patient's adherence trajectory can be identified and used to tailor interventions.

Motivational interviewing intervention tailored to patients' past adherence barriers is effective in improving statin adherence.

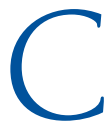
ardiovascular disease (CVD) remains a major global cause of morbidity and mortality, affecting $40 \%$ of women and $50 \%$ of men over their lifetime. ${ }^{1,2}$ CVD is also a leading contributor to disease burden in adults aged 60 years and older, accounting for around 30\% of the total burden. ${ }^{3}$ Statin medications are the most widely used lipidlowering agents, with compelling clinical trial data demonstrating effectiveness in primary and secondary prevention of CVD as well as evidence showing significant reduction of cardiovascular-related mortality.-10 Despite these well-documented benefits, adherence and persistence to statins remain substandard.,11 High discontinuation rates have also been reported, with $50 \%$ of patients discontinuing within 1 year and increasing discontinuation over time, especially among elderly patients. ${ }^{4,11-15}$

In the United States, 33\%-69\% of all medication-related hospital admissions are attributable to poor medication adherence, resulting in an annual estimated cost of $\$ 100$ billion. ${ }^{16,17}$ About $40 \%-86 \%$ of older adults are nonadherent, ${ }^{17-19}$ which is concerning because they usually experience a higher number of illnesses, use more medications, and are at risk of age-related cognitive decline. ${ }^{20,21}$ 


\section{Enhancing Statin Adherence Using a Motivational Interviewing Intervention and Past Adherence Trajectories in Patients with Suboptimal Adherence}

Motivational interviewing (MoI) is a patient-centered form of counseling that influences positive health behaviors through strengthening personal motivation and promoting self-efficacy to change..$^{22-24} \mathrm{MoI}$ has been used to foster positive behavioral changes in alcohol abuse, smoking cessation, weight loss, drug misuse, and medication adherence. ${ }^{25-29}$ An Mol phone intervention by pharmacy students improved adherence to angiotensin-converting enzyme inhibitors or angiotensin receptor blockers among nonadherent Medicare Advantage patients with comorbid hypertension and diabetes. ${ }^{27}$ The study identified nonadherent patients by examining 6-month refill claims data using the medication proportion of days covered (PDC) calculation; $<0.8(<80 \%)$ was considered nonadherent.

PDC is frequently used as a measure of patient adherence, and patients with $80 \%$ or higher PDC are considered adherent. ${ }^{30-32}$ The relationship between statin PDC and clinical outcomes has been demonstrated in research using administrative claims data. ${ }^{32,33}$ One limitation of the PDC measure is that it provides a single number to represent a follow-up period; thus, patients with variable adherence patterns can have similar PDC values. ${ }^{31,32}$ The variations in patterns of patients' adherence to statins are strongly associated with subsequent cardiovascular events, can influence patient health outcomes, and should be considered when designing adherence interventions. ${ }^{31,32}$ Group-based trajectory models (GBTM) designed to identify patients with similar longitudinal patterns can summarize adherence while capturing its dynamic nature. ${ }^{31}$ Visual patterns of adherence over time provide a qualitative dimension that can better inform intervention development and enhance intervention effectiveness.

Therefore, the objective of this study was to evaluate the effectiveness of an Mol pharmacy student phone intervention in improving adherence to statin medications among nonadherent patients enrolled in a Medicare Advantage prescription drug plan (MAPD). The intervention was customized by patients' past 1-year adherence patterns and identified through trajectory modeling, thus allowing for a more tailored use of MoI strategies.

\section{Methods}

\section{Study Design}

This was a prospective study conducted in 2017 to provide a phone-based MoI intervention to nonadherent patients enrolled in a Texas-based MAPD. Nonadherent patients from the plan were identified retrospectively using administrative claims data. ${ }^{34}$ Phase 2 of the study was to conduct an MoI intervention using pharmacy students in a sample of the identified patients and follow the patients over 6 months to identify improvement in statin adherence.

\section{Identification of Candidates for Mol Intervention}

Patients using a statin were retrospectively identified in phase 1 of the study by examining pharmacy and medical claims data from the MAPD. ${ }^{34}$ Patients were required to have (a) a statin prescription between January 2015 and June 2015 and (b) continuous enrollment for 2 years before and 1 year following the identified statin prescription. Patients could be either new or prevalent users of statins at their identification in 2015. The study intended to focus on examining patterns of statin adherence; therefore, all statin users were included regardless of the indication of statin use. There were 7,850 patients who met these criteria, and their adherence was measured for 1 year following their identification using pharmacy refill data. GBTM was used to identify 4 distinct adherence groups. Description of the modeling and identification as well as visual representation of the groups are published elsewhere. ${ }^{34}$ The 4 groups obtained from the analysis were (a) rapid decline or discontinuation, (b) gradually declining adherence over time, (c) gaps in adherence, and (d) high or nearly perfect adherence.

The flowchart for patient selection is presented in Figure 1. Only the first 3 groups, comprising 3,282 eligible patients with lower adherence, were considered as potential candidates for the prospective intervention. They were initially randomized into the intervention and control groups in a 1:1 manner with 1,641 patients set aside for the potential control group and the remaining 1,641 assigned as candidates for intervention. Patients selected to receive the intervention were then contacted by phone during the implementation phase until a sample size of 150 participants was reached. Two controls for each patient who received the intervention were then selected randomly (1:2) from the potential control group of 1,641 patients, and a sample of 450 was estimated to be used for the outcome assessment. The sample size calculation was performed using G-Power 3.1, and a total of 409 patients were estimated for a 2-tailed analysis using logistic regression at a 0.05 a-level, 0.10 $\beta$-level (90\% power), and for an expected 1.5 odds ratio, which was based on previous research. ${ }^{27,35}$

\section{Mol Training and Intervention Material}

MoI scripts and cases for role play were created by MoI-trained faculty and reviewed by an external certified Mol trainer. The scripts were templates to help guide students through the phone interview process and were customized to each adherence trajectory determined from the retrospective phase (rapid decline, gradual decline, and gaps in adherence). The information in the scripts was obtained from extensive literature evaluation on both MoI and barriers to statin adherence. ${ }^{36}$ The GBTM analysis also identified patient predictors of being in each adherence trajectory to understand differences among patients in each group. From the available information, it was hypothesized that patients with rapid decline would be more likely to have side effects or fear of side effects, cost, and 


\section{Enhancing Statin Adherence Using a Motivational Interviewing Intervention and Past Adherence Trajectories in Patients with Suboptimal Adherence}

\section{FIGURE 1 Flowchart for Patient Selection}

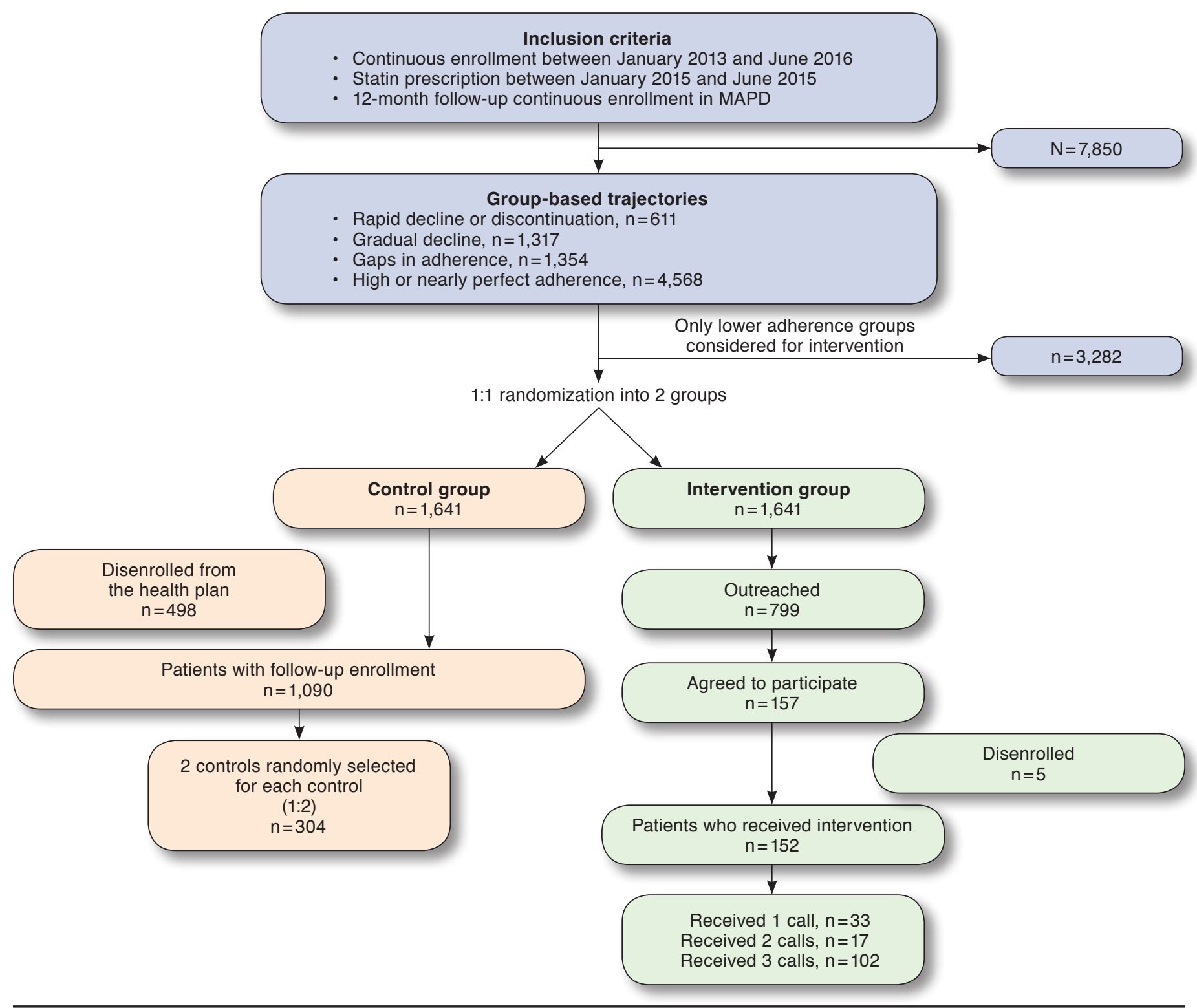

MAPD $=$ Medicare Advantage prescription drug plan.

transportation issues. Patients in the other groups would be more likely to have forgetfulness barriers or a perception that the statin is not needed anymore. A list of potential barriers hypothesized within each trajectory and possible solutions were listed within the MoI scripts to help the students reflect patient barriers in an MoI-consistent manner. In addition to the barriers, the script included a general outline based on the ask-provide-ask approach and can be available on request. ${ }^{37}$
The MoI was conducted by pharmacy students either as part of an MoI elective or a 6-week advanced pharmacy practice experiential rotation. Students received MoI training at a college of pharmacy by a trainer who is a member of the Motivational Interviewing Network of Trainers. The training was provided over a 2-day session. The in-class training involved lectures, discussion, videos, demonstrations, and role-playing. The students practiced cases with trained standardized patients and were evaluated by the trainers for using 


\section{Enhancing Statin Adherence Using a Motivational Interviewing Intervention and Past Adherence Trajectories in Patients with Suboptimal Adherence}

MoI skills and received feedback. Approximately 25 students participated in the training and conducted the intervention.

\section{Intervention}

The intervention was scheduled to be held between January and September 2017. The intervention consisted of 1 initial call followed by 2 follow-up phone calls each spaced a month apart. The recruitment and initial MoI phone calls were placed between January to June 2017, and the second and third followup calls were conducted until September 2017. The students were supervised by preceptors and residents at the MAPD location. Further, students were provided a list of patients along with their basic demographic information, current prescription medications, and their last refill dates. Students attempted contact with patients even if the patients did not have a statin refill in the past 6 months or greater, because it could not be confirmed if this observed statin discontinuation in claims was because of patient nonadherence or a change in drug by the physician. Patients who did not respond to phone calls after 3 attempts were considered nonresponders. The initial call included obtaining informed consent from the patient, and if the patient consented to receive the intervention, an MoI-consistent interaction between the student and patient also occurred during the same call. The date of the call was identified as the study index date. The student confirmed the diagnosis, medication name, and dosage with the patient and then initiated a discussion using the appropriate script based on the patient's adherence trajectory. The intervention was provided by the students in English. All information collected at the MAPD location was de-identified and provided to the University of Houston for further analysis. The intervention and data collection protocol was approved by the University of Houston Institutional Review Board.

\section{Outcome Measures}

The date of the initial call was identified as the index date for the intervention group. The corresponding controls for each patient were assigned an index date as the first of the same month to ensure that the follow-up time for the controls and intervention patients was the same. As the effect of the MoI on patient adherence was assumed to start immediately after the initial call, the follow-up for outcomes was started from the date of the first call. All patients were followed and required to be continuously enrolled for 180 days after the index date to measure adherence to statins as PDC using their pharmacy refill data from the MAPD. The effect of the intervention was assessed on the following outcomes: (a) adherence in the follow-up assessed as a binary measure, with a PDC $\geq 0.8$ considered as adherent and $<0.8$ considered nonadherent; (b) adherence in the follow-up as a continuous measure, which was calculated as the PDC value multiplied by 100 to give a continuous scale between 0 and 100; and (c) discontinuation in the follow-up defined as no record of statin refills in the entire follow-up period.

\section{Other Variable Definitions}

Baseline and clinical characteristics that were measured from the administrative claims included age, sex, preferred language of the patient (English or Spanish), presence of low-income subsidy (LIS) indicating additional financial assistance in paying for prescriptions, physician specialty (primary care practitioner vs. specialist), Deyo adaptation of the Charlson Comorbidity Index score and Centers for Medicare \& Medicaid Services (CMS) risk score. ${ }^{38}$ The CMS risk score consists of 189 disease classifications for use in risk adjusting of clinical outcomes in Medicare populations and can be reflective of patient severity. ${ }^{39,40}$ Presence of comorbidities including hyperlipidemia, hypertension, congestive heart failure, and diabetes were also independently assessed. Use of a statin for primary versus secondary prevention was adjusted in the model. Hospitalization in the 6 months before the index date was also measured. Adherence trajectories obtained from the GBTM provided an estimation of patients' baseline adherence pattern. These characteristics were added to all multivariable analytical models for outcome assessment.

All patients had a 1-year adherence measurement before the intervention; therefore, none of the patients were statin-naive. Very few $(0.82 \%)$ patients had mail order pharmacy during the retrospective phase, so it was not a variable added to the multivariable model.

\section{Statistical Analysis}

Baseline and clinical characteristics were compared between the intervention and control group. Additionally, among the intervention group, the baseline characteristics were also compared between patients who agreed to participate in the intervention as compared with those who declined or could not be reached. T-tests were used for continuous variables and chi-square tests for categorical variables. To evaluate the effect of MoI on 6-month follow-up adherence, the outcome PDC was evaluated as a binary variable (PDC $\geq 0.80$ vs. not), continuous variable (0-100), and complete discontinuation $(P D C=0)$. A logistic multivariable regression model was used to model $P D C \geq 0.80$ as the outcome variable and intervention versus the control group as the primary independent variable while controlling for all the baseline clinical and demographic variables mentioned above. A multiple linear regression was modeled with PDC as the outcome. Finally, logistic regression was performed to the association between the intervention and complete discontinuation in the 180-day follow-up. Interaction was assessed between the variables, which had a significant association with the outcome in each model. All statistical analyses were performed using SAS software version 9.4 (SAS Institute, Cary, NC) at a priori significance level of 0.05 . 


\section{Enhancing Statin Adherence Using a Motivational Interviewing Intervention and Past Adherence Trajectories in Patients with Suboptimal Adherence}

\begin{tabular}{|c|c|c|c|}
\hline Variable & $\begin{array}{c}\text { Intervention Group } \\
(\mathrm{n}=152)\end{array}$ & $\begin{array}{c}\text { Control Group } \\
(\mathrm{n}=304)\end{array}$ & $P$ Value \\
\hline \multicolumn{4}{|l|}{ Trajectory, n (\%) } \\
\hline Rapid decline & $24 \quad(15.8)$ & $55 \quad(18.1)$ & \multirow{3}{*}{0.729} \\
\hline Gradual decline & $50 \quad(32.9)$ & $104 \quad(34.2)$ & \\
\hline Gaps in adherence & $78 \quad(51.3)$ & $145 \quad(47.7)$ & \\
\hline \multicolumn{4}{|l|}{ Sex, n (\%) } \\
\hline Male & $56 \quad(36.9)$ & $126 \quad(41.4)$ & \multirow{2}{*}{0.344} \\
\hline Female & $96 \quad(63.2)$ & $178 \quad(58.6)$ & \\
\hline \multicolumn{4}{|l|}{ Age, n (\%) } \\
\hline$<65$ & $25 \quad(16.5)$ & $53 \quad(17.4)$ & \multirow{4}{*}{0.774} \\
\hline $65-70$ & $58 \quad(38.2)$ & $111 \quad(36.5)$ & \\
\hline $71-75$ & $40 \quad(26.3)$ & $71 \quad(23.4)$ & \\
\hline$>75$ & $29 \quad(19.1)$ & $69 \quad(22.7)$ & \\
\hline \multicolumn{4}{|l|}{ Preferred language, n (\%) } \\
\hline English & $150 \quad(98.7)$ & $208 \quad(68.4)$ & \multirow{2}{*}{$<0.001$} \\
\hline Spanish & $(1.3)$ & $96 \quad(31.6)$ & \\
\hline \multicolumn{4}{|c|}{ Low-income subsidy, n (\%) } \\
\hline No & $90 \quad(59.2)$ & $142 \quad(46.7)$ & \multirow{2}{*}{0.012} \\
\hline Yes & $62 \quad(40.8)$ & $162 \quad(53.3)$ & \\
\hline \multicolumn{4}{|l|}{ Physician specialty, n (\%) } \\
\hline Primary care provider & $113 \quad(74.3)$ & $235 \quad(77.6)$ & \multirow{2}{*}{0.446} \\
\hline Specialist & $39 \quad(25.7)$ & $68 \quad(22.4)$ & \\
\hline \multicolumn{4}{|l|}{ Hyperlipidemia, n (\%) } \\
\hline No & $(9.2)$ & $(5.9)$ & \multirow{2}{*}{0.195} \\
\hline Yes & $138 \quad(90.8)$ & $286 \quad(94.1)$ & \\
\hline \multicolumn{4}{|l|}{ Hypertension, n (\%) } \\
\hline No & $10 \quad(6.6)$ & $48 \quad(15.8)$ & \multirow{2}{*}{0.005} \\
\hline Yes & $142 \quad(93.4)$ & $256 \quad(84.2)$ & \\
\hline \multicolumn{4}{|l|}{ Diabetes, n (\%) } \\
\hline No & $74 \quad(48.7)$ & $131 \quad(43.1)$ & \multirow{2}{*}{0.258} \\
\hline Yes & $78 \quad(51.3)$ & $173 \quad(56.9)$ & \\
\hline \multicolumn{4}{|c|}{ Statin use for secondary prevention, n (\%) } \\
\hline No & $12 \quad(7.9)$ & $13 \quad(4.3)$ & \multirow{2}{*}{0.110} \\
\hline Yes & $140 \quad(92.1)$ & $291 \quad(95.7)$ & \\
\hline \multicolumn{4}{|l|}{ Baseline PDC } \\
\hline Mean \pm SD & $0.61 \pm 0.20$ & $0.56 \pm 0.21$ & 0.019 \\
\hline \multicolumn{4}{|c|}{ Outcomes of interest in the follow-up } \\
\hline \multicolumn{4}{|l|}{ PDC in the follow-up } \\
\hline Mean \pm SD & $0.67 \pm 0.31$ & $0.55 \pm 0.36$ & $<0.001$ \\
\hline \multicolumn{4}{|c|}{ PDC $\geq 0.8$ in the follow-up, $\mathrm{n}(\%)$} \\
\hline No & $81 \quad(53.3)$ & $202 \quad(66.5)$ & \multirow{2}{*}{0.006} \\
\hline Yes & $71 \quad(46.7)$ & $102 \quad(33.5)$ & \\
\hline Statin discontinuation & in the follow-up, n (\%) & & \\
\hline No & $137 \quad(90.3)$ & $242 \quad(79.6)$ & 0005 \\
\hline Yes & (9.9) & $62 \quad(20.4)$ & 0.003 \\
\hline
\end{tabular}

\section{Results}

Identification of Intervention Candidates, Mol Intervention, and Baseline Characteristics

The demographic and baseline clinical characteristics of the patients assigned to the intervention and control group were assessed and no differences were found between them. The flowchart of patient selection is presented in Figure 1. Of the 1,641 patients in the intervention group, 799 patients were outreached at least once between January and June 2017. Participation in the intervention was accepted by 157 patients. There were no differences in the demographic characteristics analyzed using chi-square and t-tests, including age, sex, LIS, and physician specialty, between the patients who agreed to participate as compared with those who declined or did not answer the phone call, except for their preferred language as more patients who declined participation had Spanish as their preferred language (8.6\% declined vs. $1.3 \%$ participated; results not shown). Furthermore, only 152 patients were continuously enrolled for 6 months for follow-up adherence assessment, since pharmacy claims data for the patients disenrolled from the plan would not be available for adherence measurement. All 3 calls were completed for 102 patients, while 17 patients completed only 2 calls, and 33 patients participated only in the first call.

Of the patients randomized to the control group, 1,090 patients were still enrolled in the MAPD during the intervention period. As there were 2 patients randomly selected from the control group for each patient in the intervention group (1:2), there were 304 final controls. Table 1 presents the baseline characteristics across the 2 groups. More patients in the intervention group had English as their preferred language (98.7\%) as compared with the control group (68.4\%). A smaller proportion of patients had a LIS in the intervention group as compared with the control group, and the baseline PDC in the intervention group was higher than the control group. There was no significant difference across any of the other demographic variables between the 2 groups.

Table 1 also shows the mean PDC, categorized PDC $(\geq 0.8)$, and discontinuations in the 180-day follow-up across the 2 groups. The rapid decline group had the lowest adherence in both the intervention and control groups with an improvement in both groups at follow-up. The baseline and post-intervention PDC across each of the trajectories are presented in Table 2. The gradual decline and gaps in adherence trajectories had higher mean adherence than the rapid decline trajectory on both pre- and post-intervention periods.

\section{Results of Multivariable Models for Outcome Assessment}

Dichotomous Outcome for Adherence: $P D C \geq 0.8$ in the Follow-Up. Results from the multivariable logistic regression model evaluating the association between $\mathrm{MoI}$ intervention and adherence in the follow-up are presented in Table 3. Patients 
Enhancing Statin Adherence Using a Motivational Interviewing Intervention

and Past Adherence Trajectories in Patients with Suboptimal Adherence

TABLE 2 Mean Proportion of Days Covered Across Trajectories at Baseline and in the 180-Day Follow-Up

\begin{tabular}{|c|c|c|c|c|}
\hline \multirow[b]{2}{*}{ Proportion of Days Covered } & \multicolumn{2}{|c|}{ Intervention Group $(\mathrm{n}=152)$} & \multicolumn{2}{|c|}{ Control Group $(n=304)$} \\
\hline & $\begin{array}{l}\text { Baseline } \\
\text { Mean (SD) }\end{array}$ & $\begin{array}{c}\text { Post-Intervention } \\
\text { Mean (SD) }\end{array}$ & $\begin{array}{l}\text { Baseline } \\
\text { Mean (SD) }\end{array}$ & $\begin{array}{c}\text { Post-Intervention } \\
\text { Mean (SD) }\end{array}$ \\
\hline \multicolumn{5}{|l|}{ Trajectory } \\
\hline Rapid decline $(\mathrm{n}=79)$ & $0.25 \quad(0.10)$ & $(0.42)$ & $0.23 \quad(0.07)$ & $(0.34)$ \\
\hline Gradual decline $(n=154)$ & $0.69 \quad(0.12)$ & $(0.30)$ & $0.67 \quad(0.13)$ & $0.52 \quad(0.37)$ \\
\hline Gaps in adherence $(n=223)$ & $0.67 \quad(0.14)$ & $(0.26)$ & $0.62 \quad(0.15)$ & $0.66 \quad(0.30)$ \\
\hline
\end{tabular}

receiving the intervention were more likely to be adherent in the follow-up as compared with the control group (adjusted odds ratio $[\mathrm{OR}]=1.87,95 \%$ confidence interval $[\mathrm{CI}]=1.18$ 2.95). Patients who were in the gradual decline or gaps in adherence trajectory at baseline were more likely to be adherent in the follow-up as compared with patients in the rapid decline trajectory. Patients having a LIS were associated with lower adherence (adjusted $\mathrm{OR}=0.6,95 \% \mathrm{CI}=0.4-0.9$ ). Patients with an increasing CMS score were associated with an increased likelihood of being adherent (adjusted OR $=1.4,95 \%$ $\mathrm{CI}=1.1-1.8)$

Continuous Measure of Adherence. The intervention group has a significantly higher mean $\mathrm{PDC}(0.67 \pm 0.31)$ as compared with the control group $(0.55 \pm 0.36 ; P<0.001)$ as seen in Table 1 . This relationship was preserved in the multiple regression model where the patients in the intervention group had a higher mean PDC $(\beta=12.39, P=0.0004)$ than the controls when adjusting for baseline trajectories and all relevant clinical and demographic characteristics as presented in Table 3. Similar to the logistic regression model, patients in the gradual decline and gaps in adherence group had a higher mean adherence than the rapid decline group. Patients with higher CMS scores had higher mean adherence $(\beta=4.77, P=0.022)$, and patients with LIS had lower mean adherence $(\beta=-7.62$, $P=0.023)$. There was no multicollinearity observed among the added covariates

Discontinuation of Statins. As shown in Table 1, a significantly greater proportion of patients in the control group (20.4\%) had discontinued statins as compared with the intervention group (9.9\%). Results from the multivariable logistic regression adjusting for adherence trajectory, demographics, and clinical variables are available in Table 4. Patients who received the intervention were less likely to discontinue statins (adjusted $\mathrm{OR}=0.38,95 \%$ $\mathrm{CI}=0.19-0.76$ ). The gradual decline (adjusted $\mathrm{OR}=0.24$, $95 \% \mathrm{CI}=0.12-0.46)$ and gaps in adherence (adjusted $\mathrm{OR}=0.10,95 \%$ $\mathrm{CI}=0.05-0.20)$ trajectory groups were less likely to discontinue as compared with the rapid decline group.

\section{Discussion}

Adherence is a pivotal link between medication prescribing and treatment success and is necessary for achieving full medication effectiveness leading to more favorable health outcomes. ${ }^{17,18,21}$ With over 32 million Americans reporting to have prescription drug benefits from Medicare (through Medicare Part D or Medicare Advantage), ${ }^{41}$ and given the positive effect of statins in reducing risk in CVD when taken as prescribed, ${ }^{5-10}$ effective interventions to improve statin adherence among older adults are greatly needed and can translate into significant health improvements and overall cost savings.

Findings of this study present a promising tailored intervention to enhance statin adherence among nonadherent patients enrolled in an MAPD. Compared with controls, patients who received the intervention had significantly less discontinuation and better adherence rates measured during the 6-month postintervention follow-up. The intervention was phone-based, tailored according to past adherence patterns, and implemented by pharmacy students who were trained to use Mol strategies. A previous study that examined the effectiveness of an MoI-based phone intervention conducted by pharmacy students for nonadherent patients has established the feasibility of training pharmacy students in MoI as well as conducting a phone-based intervention to nonadherent patients. ${ }^{27}$ The study demonstrated an improvement in the post-intervention 6-month adherence among patients completing at least 3 calls. ${ }^{27}$ MoI strategies have been shown to improve medication adherence in a number of chronic illnesses including asthma, ${ }^{28}$ hypertension, and human immunodeficiency virus. ${ }^{29,42}$ Phone-based interventions can incorporate the use of MoI, and trained students can be a cost-efficient way to deliver such interventions..$^{27,43-45}$

This study was unique as the intervention implemented was also tailored by the patients' past adherence patterns. Trajectory modeling was used to identify patients with specific patterns of adherence, providing a more qualitative dimension to patient behavior as patients with the same PDC could have different trajectories of adherence. ${ }^{31,34}$ Identified trajectories among the nonadherent patients included rapid decline or 


\section{Enhancing Statin Adherence Using a Motivational Interviewing Intervention and Past Adherence Trajectories in Patients with Suboptimal Adherence}

TABLE 3 Result of Regression Models to Examine Effect of Intervention on Adherence

\begin{tabular}{|c|c|c|c|c|c|c|}
\hline \multirow{3}{*}{$\frac{\text { Variable }}{\text { Intervention vs. control group }}$} & \multicolumn{3}{|c|}{ Model 1: Logistic Regression } & \multicolumn{3}{|c|}{ Model 2: Linear Regression } \\
\hline & \multicolumn{2}{|c|}{$\begin{array}{c}\text { Adjusted Odds Ratio } \\
(95 \% \mathrm{CI})\end{array}$} & \multirow{2}{*}{$\frac{P \text { Value }}{0.008}$} & \multicolumn{2}{|c|}{$\beta$ Estimate (SE) } & \multirow{2}{*}{$\frac{P \text { Value }}{<0.001}$} \\
\hline & 1.87 & $(1.18-2.95)$ & & 12.40 & $(3.47)$ & \\
\hline \multicolumn{7}{|l|}{ Trajectory } \\
\hline Gradual decline vs. rapid decline & 3.15 & $(1.59-6.25)$ & 0.001 & 23.20 & $(4.52)$ & $<0.001$ \\
\hline Gaps in adherence vs. rapid decline & 3.74 & $(1.94-7.21)$ & $<0.001$ & 32.07 & $(4.29)$ & $<0.001$ \\
\hline \multicolumn{7}{|l|}{ Sex } \\
\hline Female vs. male & 0.98 & $(0.64-1.49)$ & 0.905 & -0.39 & (3.19) & 0.902 \\
\hline \multicolumn{7}{|l|}{ Age } \\
\hline $65-70$ vs. $<65$ & 1.24 & $(0.66-2.33)$ & 0.505 & 0.54 & $(4.70)$ & 0.505 \\
\hline $71-75$ vs. $<65$ & 1.25 & $(0.64-2.43)$ & 0.514 & 1.24 & $(5.00)$ & 0.514 \\
\hline$>75$ vs. $<65$ & 0.89 & $(0.45-1.77)$ & 0.746 & -4.55 & $(5.00)$ & 0.746 \\
\hline \multicolumn{7}{|l|}{ Preferred language } \\
\hline Spanish vs. English & 1.48 & $(0.87-2.55)$ & 0.152 & 6.57 & $(4.09)$ & 0.110 \\
\hline \multicolumn{7}{|l|}{ Low-income subsidy } \\
\hline Having subsidy vs. not & 0.59 & $(0.38-0.92)$ & 0.021 & -7.62 & (3.33) & 0.023 \\
\hline \multicolumn{7}{|l|}{ Physician specialty } \\
\hline Specialist vs. primary care provider & 0.93 & $(0.58-1.50)$ & 0.764 & 0.19 & (3.63) & 0.957 \\
\hline CMS risk score & 1.38 & $(1.05-1.82)$ & 0.022 & 4.77 & $(2.08)$ & 0.022 \\
\hline Charlson Comorbidity Index score & 0.74 & $(0.52-1.05)$ & 0.091 & -4.29 & $(2.36)$ & 0.070 \\
\hline Diabetes (yes vs. no) & 1.22 & $(0.79-1.90)$ & 0.369 & 4.16 & $(3.32)$ & 0.210 \\
\hline Hyperlipidemia (yes vs. no) & 1.92 & $(0.26-14.39)$ & 0.527 & 12.09 & $(12.74)$ & 0.343 \\
\hline Hypertension (yes vs. no) & 1.09 & $(0.58-2.08)$ & 0.783 & 3.29 & $(4.75)$ & 0.489 \\
\hline CHF (yes vs. no) & 0.96 & $(0.44-2.11)$ & 0.915 & -4.77 & $(5.98)$ & 0.426 \\
\hline Statin use for secondary vs. primary prevention & 0.77 & $(0.09-6.94)$ & 0.819 & -8.49 & $(14.29)$ & 0.553 \\
\hline Hospitalized in past 6 months vs. not & 0.93 & $(0.46-1.90)$ & 0.850 & 0.43 & $(5.38)$ & 0.937 \\
\hline
\end{tabular}

discontinuation, gradual decline over time, and gaps in adherence that improved over time.

The potential barriers likely to be associated with each trajectory, and strategies that can help patients within each trajectory, were based on patients' identified behavior pattern. In a previously conducted study, the intervention was effective only in the subset of patients who had received 3 or more calls, and no significant difference was found in the overall group. ${ }^{27}$ In this study, a significant difference was seen in the post-intervention adherence when comparing intervention patients with controls, even when including the patients who completed the initial call only. Knowledge of the past adherence patterns may potentially enhance the $\mathrm{MoI}$ intervention effectiveness through providing more insight into patient adherence behavior and potential barriers, thus allowing a more productive conversation and a better connection with the patient.

Additionally, results showed that patients with the gradual decline trajectory and the gaps in adherence trajectory exhibited significantly higher mean adherence and less discontinuation during the 6 months following the initial intervention call compared with patients in the rapid decline trajectory. These patients had a lower absolute mean PDC compared with the other trajectories before the intervention and in the follow-up, but the improvement in magnitude was also highest among these patients. The lower adherence as compared with the other groups may indicate that the barriers potentially associated with rapid decline trajectory may be out of patient control such as side effects. On the other hand, it could also indicate that the consistent poor adherence was a result of patient beliefs including need of the medication. More follow-up calls may also be needed for a patient who may view the medication as unnecessary. Future research is needed to fully ascertain differences in barriers within different trajectories and the best ways to address them as well as the number of follow-up calls that may be needed for patients with variable trajectories.

Other predictors of adherence included CMS risk score and LIS status in the health plan. Having a higher risk score was associated with improved adherence after controlling for potential confounders. Literature has been inconsistent with regards to the effect of comorbidities and severity of illness on medication adherence. Some studies report that having a more severe case or more comorbidities that complicate the medication regimen can negatively influence adherence, and others report that sicker patients may be more attentive to their 


\section{Enhancing Statin Adherence Using a Motivational Interviewing Intervention and Past Adherence Trajectories in Patients with Suboptimal Adherence}

\begin{tabular}{|c|c|c|}
\hline $\begin{aligned} \text { TABLE } 4 & \text { Result of Log } \\
& \text { Examine Eff } \\
& \text { Discontinuat }\end{aligned}$ & $\begin{array}{l}\text { istic Regression } \Lambda \\
\text { ct of Intervention } \\
\text { on }\end{array}$ & $\begin{array}{l}\text { del to } \\
\text { on Statin }\end{array}$ \\
\hline Variable & $\begin{array}{c}\text { Adjusted Odds Ratio } \\
(95 \% \mathrm{CI})\end{array}$ & $P$ Value \\
\hline Intervention vs. control group & $0.38(0.19-0.76)$ & 0.006 \\
\hline \multicolumn{3}{|l|}{ Trajectory } \\
\hline Gradual decline vs. rapid decline & $0.24(0.12-0.46)$ & $<0.001$ \\
\hline Gaps in adherence vs. rapid decline & $0.10 \quad(0.05-0.20)$ & $<0.001$ \\
\hline \multicolumn{3}{|l|}{ Sex } \\
\hline Female vs. male & $0.84(0.48-1.48)$ & 0.545 \\
\hline \multicolumn{3}{|l|}{ Age } \\
\hline $65-70$ vs. $<65$ & $0.98 \quad(0.42-2.29)$ & 0.961 \\
\hline $71-75$ vs. $<65$ & $0.70 \quad(0.27-1.78)$ & 0.444 \\
\hline$>75$ vs. $<65$ & $1.71 \quad(0.73-4.04)$ & 0.219 \\
\hline \multicolumn{3}{|l|}{ Preferred language } \\
\hline Spanish vs. English & $0.79 \quad(0.39-1.61)$ & 0.519 \\
\hline \multicolumn{3}{|l|}{ Low-income subsidy } \\
\hline Having subsidy vs. not & $1.01 \quad(0.55-1.83)$ & 0.988 \\
\hline \multicolumn{3}{|l|}{ Physician specialty } \\
\hline Specialist vs. primary care provider & $0.83(0.42-1.63)$ & 0.586 \\
\hline CMS risk score & $0.74 \quad(0.48-1.15)$ & 0.184 \\
\hline Charlson Comorbidity Index score & $1.36(0.96-1.93)$ & 0.082 \\
\hline Diabetes (yes vs. no) & $0.67 \quad(0.37-1.21)$ & 0.184 \\
\hline Hyperlipidemia (yes vs. no) & $0.34(0.05-2.39)$ & 0.280 \\
\hline Hypertension (yes vs. no) & $0.79 \quad(0.36-1.73)$ & 0.561 \\
\hline CHF (yes vs. no) & $1.01 \quad(0.32-3.19)$ & 0.982 \\
\hline $\begin{array}{l}\text { Statin use for secondary vs. primary } \\
\text { prevention }\end{array}$ & $3.62(0.35-37.35)$ & 0.280 \\
\hline Hospitalized in past 6 months vs. not & $1.08(0.39-2.96)$ & 0.883 \\
\hline
\end{tabular}

medications showing better adherence, which is consistent with our findings. ${ }^{12,14,46,47}$

LIS was associated with lower adherence levels during the 6 months following intervention implementation. Patients have to meet certain income eligibility criteria to qualify for LIS, and socioeconomic factors are known to lower adherence. ${ }^{47,48}$ The retrospective phase of the study had also found that patients with LIS were more likely to fall into lower adherence trajectories. As this difference was still observed after the intervention, it highlights that future interventions should consider such factors and their effect on medication adherence to further tailor the intervention conversation to patients' specific needs, expectations, and barriers.

\section{Limitations}

This study has several limitations to be considered. While adherence refill rates are widely used as a measure of overall adherence, prescription filling does not always guarantee that the patient has taken the medication as prescribed. ${ }^{16,21}$ Samples or prescriptions paid for by cash cannot be verified. This study initially randomized nonadherent patients to intervention and control groups, but many patients contacted for the intervention group did not answer the call or consent to the study, resulting in the eventual loss of randomization. Differences in baseline characteristics, however, were controlled for in multivariable analysis. The baseline characteristics between patients with successful calls and the group of patients attempted to be reached but without successful calls were also compared with preferred language being the only significant difference between the 2 groups.

Differences in other factors may exist between the patients who consented to the calls and those who were not willing to participate and can only be addressed using a fully randomized design. Since the intervention was provided in English, patients preferring Spanish did not participate in the intervention. We also found differences in patients with LIS in the intervention and control groups. Although these differences were controlled in the multivariable analysis, patients may have different health behaviors and barriers to adherence and may need further evaluation in future studies. Additional potential confounders, such as side effects, health literacy, perceived health, and hospitalizations during a follow-up period, could not be controlled for in the analysis. Because of the asymptomatic nature of the hyperlipidemia and the side effect profile of statins, these confounders could affect the results or their interpretation.

Also, there was a temporal difference between the trajectory modeling and the actual intervention implementation. The effectiveness of MoI interventions can also be influenced by several other factors, such as length and number of intervention sessions, MoI delivery mode, and the MoI counselor credentials. ${ }^{49}$ The follow-up for adherence measurement was only 6 months and longer periods may be required to further assess intervention effectiveness.

\section{Conclusions}

The findings of this study present a promising intervention to enhance statin adherence among nonadherent patients. Recognizing patients' past adherence patterns provides more insight into potential barriers for each patient and, when combined with MoI, allow a more influential conversation and a better connection with the patient. A tailored approach may enhance the effectiveness of interventions to better adherence. Future research should examine the intervention effect for longer time periods, as well as new approaches to tailoring intervention to better suit patient needs. The intervention could be expanded to multiple languages to increase the number of nonadherent patients in the MAPD who can receive it, as well as focus on specific subpopulations such as patients from lower socioeconomic status. 


\section{Enhancing Statin Adherence Using a Motivational Interviewing Intervention and Past Adherence Trajectories in Patients with Suboptimal Adherence}

\section{Authors}

SUSAN M. ABUGHOSH, PhD; AISHA VADHARIYA, PhD;

MICHAEL L. JOHNSON, PhD; and E. JAMES ESSIEN, MD,

DrPH, Department of Pharmaceutical Health Outcomes and Policy,

University of Houston College of Pharmacy, Houston, Texas. TARA

W. ESSE, PharmD, BCACP; OMAR SERNA, PharmD, BCACP; and ESTEBAN GALLARDO, PharmD, CareAllies, Houston, Texas. SUSAN H. BOKLAGE, MS, MPH, Health Economics and Outcomes Research, Regeneron Pharmaceuticals, Terrytown, New York, and JEANNIE CHOI, PharmD, Health Economics and Value Assessment, Sanofi, Bridgewater, New Jersey. MARCIA M. HOLSTAD, PhD, FNP-BC, FAAN, Nell Hodgson Woodruff School of Nursing, Emory University, Atlanta, Georgia, and MARC L. FLEMING, PhD, MPH, $R P h$, Department of Pharmacotherapy, University of North Texas System College of Pharmacy, Dallas.

AUTHOR CORRESPONDENCE: Susan M. Abughosh, PhD, Associate Professor, Department of Pharmaceutical Health Outcomes and Policy, University of Houston College of Pharmacy, 4849 Calhoun Rd., Ste. 4050, Houston, TX 77204.

Tel.: 832.842.8395; E-mail: smabugho@central.uh.edu.

\section{DISCLOSURES}

This study was funded by Regeneron Pharmaceuticals, which provided critical input during study design, implementation, and manuscript preparation. Abughosh reports grants from Sanofi, BMS/Pfizer, and Valeant Pharmaceuticals, unrelated to this study. Vadhariya reports a past internship at Regeneron Pharmaceuticals, unrelated to this study. Esse, Serna, and Gallardo are employees of CareAllies, a Cigna subsidiary. Boklage is an employee of Regeneron Pharmaceuticals. Choi was an employee of Sanofi during this study. Johnson, Essien, Fleming, and Holstad have nothing to disclose.

A poster based on this study was presented at AMCP Nexus 2018; October 22-25, 2018; Orlando, FL.

\section{REFERENCES}

1. Lopez-Jaramillo P, Lopez-Lopez J, Lopez-Lopez C, Rodriguez-Alvarez MI. The goal of blood pressure in the hypertensive patient with diabetes is defined: now the challenge is go from recommendations to practice. Diabetol Metab Syndr. 2014:6(1):31.

2. Rodgers A, Ezzati M, Vander Hoorn S, Lopez AD, Lin RB, Murray CJL. Distribution of major health risks: findings from the Global Burden of Disease study. PLoS Med. 2004;1(1):e27.

3. Prince MJ, Wu F, Guo Y, et al. The burden of disease in older people and implications for health policy and practice. Lancet. 2015;385(9967):549-62.

4. Fung V, Sinclair F, Wang H, Dailey D, Hsu J, Shaber R. Patients' perspectives on nonadherence to statin therapy: a focus-group study. Perm J. 2010;14(1):4-10.

5. Cheung BM, Lauder IJ, Lau CP, Kumana CR. Meta-analysis of large randomized controlled trials to evaluate the impact of statins on cardiovascular outcomes. Br J Clin Pharmacol. 2004;57(5):640-51.

6. Cholesterol Treatment Trialists' Collaborators, Mihaylova B, Emberson J, et al. The effects of lowering LDL cholesterol with statin therapy in people at low risk of vascular disease: meta-analysis of individual data from 27 randomised trials. Lancet. 2012;380(9841):581-90.
7. Baigent C, Keech A, Kearney PM, et al. Efficacy and safety of cholesterollowering treatment: prospective meta-analysis of data from 90,056 participants in 14 randomised trials of statins. Lancet. 2005;366(9493):1267-78.

8. Thavendiranathan P, Bagai A, Brookhart MA, Choudhry NK. Primary prevention of cardiovascular diseases with statin therapy: a meta-analysis of randomized controlled trials. Arch Intern Med. 2006;166(21):2307-13.

9. Shalev V, Chodick G, Silber H, Kokia E, Jan J, Heymann AD. Continuation of statin treatment and all-cause mortality: a population-based cohort study. Arch Intern Med. 2009;169(3):260-68.

10. Delahoy PJ, Magliano DJ, Webb K, Grobler M, Liew D. The relationship between reduction in low-density lipoprotein cholesterol by statins and reduction in risk of cardiovascular outcomes: an updated meta-analysis. Clin Ther. 2009;31(2):236-44.

11. Maningat P, Gordon BR, Breslow JL. How do we improve patient compliance and adherence to long-term statin therapy? Curr Atheroscler Rep. 2013;15(1):291.

12. Abughosh SM, Kogut SJ, Andrade SE, Larrat EP, Gurwitz JH. Persistence with lipid-lowering therapy: influence of the type of lipid-lowering agent and drug benefit plan option in elderly patients. J Manag Care Pharm. 2004;10(5):404-11. Available at: https://www.jmcp.org/doi/10.18553/ jmcp.2004.10.5.404.

13. Mann DM, Woodward M, Muntner P, Falzon L, Kronish I. Predictors of nonadherence to statins: a systematic review and meta-analysis. Ann Pharmacother. 2010;44(9):1410-21.

14. Jackevicius CA, Mamdani M, Tu JV. Adherence with statin therapy in elderly patients with and without acute coronary syndromes. JAMA. 2002;288(4):462-67.

15. Benner JS, Glynn RJ, Mogun H, Neumann PJ, Weinstein MC, Avorn J. Long-term persistence in use of statin therapy in elderly patients. JAMA. 2002;288(4):455-61.

16. Osterberg L, Blaschke T. Adherence to medication. N Engl J Med. 2005;353(5):487-97.

17. Foreman KF, Stockl KM, Le LB, et al. Impact of a text messaging pilot program on patient medication adherence. Clin Ther. 2012;34(5):1084-91.

18. Butler RJ, Davis TK, Johnson WG, Gardner HH. Effects of nonadherence with prescription drugs among older adults. Am J Manag Care. 2011;17(2):153-60.

19. Kripalani S, Yao X, Haynes RB. Interventions to enhance medication adherence in chronic medical conditions: a systematic review. Arch Intern Med. 2007;167(6):540-50.

20. Murray MD, Morrow DG, Weiner M, et al. A conceptual framework to study medication adherence in older adults. Am J Geriatr Pharmacother 2004;2(1):36-43.

21. MacLaughlin EJ, Raehl CL, Treadway AK, Sterling TL, Zoller DP, Bond CA. Assessing medication adherence in the elderly: which tools to use in clinical practice? Drugs Aging. 2005;22(3):231-55.

22. Miller WR, Rollnick S. Ten things that motivational interviewing is not. Behav Cogn Psychother. 2009;37(2):129-40.

23. Christie D, Channon S. The potential for motivational interviewing to improve outcomes in the management of diabetes and obesity in paediatric and adult populations: a clinical review. Diabetes Obes Metab. 2014;16(5):381-87.

24. Velasquez MM, von Sternberg K, Dodrill CL, Kan L. The transtheoretical model as a framework for developing substance abuse interventions. J Addictions Nursing. 2005;16(1-2):31-40.

25. Rubak S, Sandbaek A, Lauritzen T, Christensen B. Motivational interviewing: a systematic review and meta-analysis. Br J Gen Pract. 2005;55(513):305-12.

26. Ogedegbe G, Chaplin W, Schoenthaler A, et al. A practice-based trial of motivational interviewing and adherence in hypertensive African Americans. Am J Hypertens. 2008;21(10):1137-43. 


\section{Enhancing Statin Adherence Using a Motivational Interviewing Intervention and Past Adherence Trajectories in Patients with Suboptimal Adherence}

27. Abughosh S, Wang X, Serna O, et al. A motivational interviewing intervention by pharmacy students to improve medication adherence. J Manag Care Spec Pharm. 2017;23(5):549-60. Available at: https://www.jmcp.org/ doi/10.18553/jmcp.2017.23.5.549.

28. Halterman JS, Szilagyi PG, Fisher SG, et al. Randomized controlled trial to improve care for urban children with asthma: results of the school-based asthma therapy trial. Arch Pediatr Adolesc Med. 2011;165:262-68.

29. Dilorio C, McCarty F, Resnicow K, et al. Using motivational interviewing to promote adherence to antiretroviral medications: a randomized controlled study. AIDS Care. 2008;20(3):273-83.

30. Peterson AM, Nau DP, Cramer JA, Benner J, Gwadry-Sridhar F, Nichol M. A checklist for medication compliance and persistence studies using retrospective databases. Value Health. 2007;10(1):3-12.

31. Franklin JM, Shrank WH, Pakes J, et al. Group-based trajectory models: a new approach to classifying and predicting long-term medication adherence. Med Care. 2013;51(9):789-96.

32. Franklin JM, Krumme AA, Tong AY, et al. Association between trajectories of statin adherence and subsequent cardiovascular events. Pharmacoepidemiol Drug Saf. 2015;24(10):1105-13.

33. Choudhry NK, Glynn RJ, Avorn J, et al. Untangling the relationship between medication adherence and post-myocardial infarction outcomes: medication adherence and clinical outcomes. Am Heart J. 2014;167(1):51-58.e5.

34. Vadhariya A, Fleming ML, Johnson ML, et al. Group-based trajectory models to identify sociodemographic and clinical predictors of adherence patterns to statin therapy among older adults. Am Health Drug Benefits. 2019;12(4):202-11.

35. Faul F, Erdfelder E, Lang AG, Buchner A. G*Power 3: a flexible statistical power analysis program for the social, behavioral, and biomedical sciences. Behav Res Methods. 2007;39(2):175-91.

36. Vadhariya A, Abughosh SM. A review of patient related barriers to statin adherence. J Epidemiol Public Health Rev. 2017;2(3).

37. Rollnick S, Miller WR, Butler CC, Aloia MS. Motivational interviewing in health care: helping patients change behavior. COPD: J Chron Obstruct Pulmon Dis. 2008;5(3):203.
38. Deyo RA, Cherkin DC, Ciol MA. Adapting a clinical comorbidity index for use with ICD-9-CM administrative databases. J Clin Epidemiol. 1992;45(6):613-19.

39. Johnson ML, Parikh N, Kunik ME, et al. Antihypertensive drug use and the risk of dementia in patients with diabetes mellitus. Alzheimers Dement. 2012;8(5):437-44

40. Parikh NM, Morgan RO, Kunik ME, et al. Risk factors for dementia in patients over 65 with diabetes. Int J Geriatric Psychiatry. 2011;26(7):749-57.

41. Kaiser Family Foundation. An overview of the Medicare Part D prescription drug benefit. Fact sheet. October 2018. Available at: http://kff.org/medicare/fact-sheet/the-medicare-prescription-drug-benefit-fact-sheet/. Accessed August 30, 2019.

42. Holstad MM, Dilorio C, Kelley ME, Resnicow K, Sharma S. Group motivational interviewing to promote adherence to antiretroviral medications and risk reduction behaviors in HIV infected women. AIDS Behavior. 2011;15(5):885-96.

43. Turner AP, Sloan AP, Kivlahan DR, Haselkorn JK. Telephone counseling and home telehealth monitoring to improve medication adherence: results of a pilot trial among individuals with multiple sclerosis. Rehabil Psychol. 2014:59(2):136-46.

44. Abrons J, Vadala T, Miller S, Cerulli J. Encouraging safe medication disposal through student pharmacist intervention. J Am Pharm Assoc (2003). 2010;50(2):169-73.

45. Low KG, Giasson H, Connors S, Freeman D, Weiss R. Testing the effectiveness of motivational interviewing as a weight reduction strategy for obese cardiac patients: a pilot study. Int J Behav Med. 2013;20(1):77-81.

46. DiMatteo MR, Haskard KB, Williams SL. Health beliefs, disease severity, and patient adherence: a meta-analysis. Med Care. 2007:45(6):521-28.

47. Rolnick SJ, Pawloski PA, Hedblom BD, Asche SE, Bruzek RJ. Patient characteristics associated with medication adherence. Clin Med Res. 2013;11(2):54-65.

48. Chan DC, Shrank WH, Cutler D, et al. Patient, physician, and payment predictors of statin adherence. Med Care. 2010;48(3):196-202.

49. VanBuskirk KA, Wetherell JL. Motivational interviewing with primary care populations: a systematic review and meta-analysis. J Behav Med. 2014:37(4):768-80. 